\section{Kopfeinsatz beim Sport rächt sich im Alter}

Wer seinen Schädel beim Sport häufig als Rammbock benutzt, muss später mit kognitiven Defiziten und Depressionen rechnen. Ursache dafür ist offenbar eine beschleunigte Neurodegeneration.

D er ehemalige US-Nationalliga-Football-Spieler Dave Duerson ist ein besonders tragisches Beispiel für die Folgen von exzessivem Kopfeinsatz bei rauen Sportarten: Der Profi erkrankte an einer chronisch traumatischen Enzephalopathie und beging vor einem Jahr Suizid. Sein Gehirn vermachte er der Wissenschaft.

Für die noch lebenden Gehirne von 34 ehemaligen Football-Nationalspielern interessierten sich Forscher der Universität Dallas. Sie unterzogen die Ex-Profis (durchschnittliches Alter 62, im Mittel vier Gehirnerschütterungen) neuropsychiatrischen Tests sowie modernen Bild gebenden Verfahren und verglichen sie mit 20 gleich alten kognitiv gesunden Männern, die weder Football spielten noch eine Gehirnerschütterung erlitten hatten.

Vier (12\%) der Sportler zeigten ein Defizit in einem der untersuchten kognitiven Bereiche, acht $(23,5 \%)$ wiesen erste kognitive Einschränkungen (Mild Cognitive Impairment, MCI) auf und zwei (6\%) hatten bereits eine manifeste Demenz.
Nur 20 (58\%) waren kognitiv unauffällig. Jeder Vierte war depressiv, drei hatten Depressionen und kognitive Defizite, die sich nicht allein auf die Depression zurückführen ließen. Bei den kognitiven Defiziten überwogen Wortfindungsstörungen, Probleme beim Benennen von Gegenständen sowie Störungen des verbalen und visuellen Gedächtnisses.

Die Störungen korrelierten gut mit Auffälligkeiten in Hirnregionen, die für Gedächtnis sowie Wortfindung und -verarbeitung nötig sind. So zeigten sich bei der Diffusions-Tensor-Bildgebung (DTI) mikrostrukturelle Schäden der weißen Substanz im bestimmten frontalen und parietalen Kortexarealen sowie im linken Temporallappen. Diese Schäden waren bei allen Ex-Profis deutlich stärker ausgeprägt als in der Kontrollgruppe, am deutlichsten aber bei den symptomatischen Profispielern. Diese zeigten zudem bei der Bestimmung des zerebralen Blutflusses Abweichungen im Gyrus temporalis superior, also auch im Bereich des

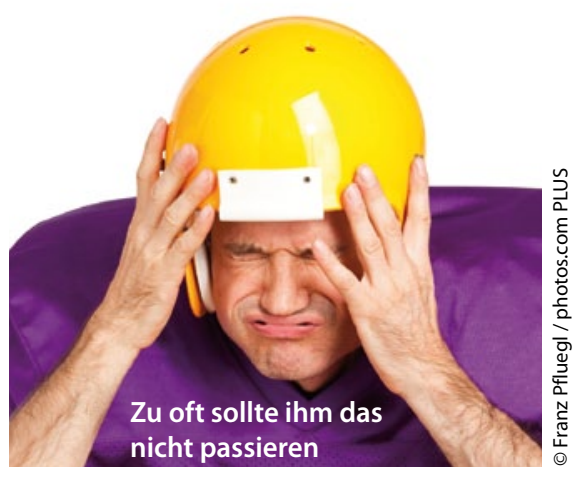

Wernicke-Zentrums, sowie im linken unteren Parietallappen.

Die Forscher vermuten, dass die kognitiven und affektiven Veränderungen durch Läsionen in der weißen Substanz und in den kortikalen Bereichen verursacht werden, die in der Bildgebung auffällig sind. Sie interpretieren die Befunde weniger als Narben vergangener Traumata, sondern vielmehr als dynamischen Prozess: Offenbar beschleunigt sich bei den ehemaligen Athleten die Neurodegeneration.

Thomas Müller

Hart J et al. Neuroimaging of Cognitive Dysfunction and Depression in Aging Retired National Football League Players A Crosssectional Study. JAMA Neurol. 2013; Epub 7.1.2013, doi:10.1001/2013.jamaneurol.340

\section{Bestimmte T-Zellen im Liquor markieren schon frühzeitig eine Multiple Sklerose}

Forscher charakterisierten bestimmte T-Zellen, die schon beim ersten MSSchub sehr spezifisch im Liquor aktiv sind und daher die Diagnosestellung verbessern und als neue Targets für MS-Therapeutika dienen könnten.

\footnotetext{
A ls Bindeglied zwischen angeborener und erworbener Immunantwort gelten sogenannte $\gamma \delta$-T-Zellen. Das Team um Dr. Lucas Schirmer, TU München, interessiert sich für diesen Zelltyp bei Patienten mit neuroinflammatorischen Erkrankungen (18 Patienten mit einem ersten klinischen MS-Schub, 8 Patienten mit einem MS-Rezidiv, 10 Patienten mit anderen Autoimmunerkrankungen, $12 \mathrm{~Pa}$ tienten mit neurologischen Infekten und 15 Patienten mit nichtentzündlichen neurologischen Krankheiten). Zunächst stellten Schirmer et al. fest, dass $\gamma \delta$-T-Zellen
}

insgesamt bei MS-Patienten sowohl im Blut $(7,4 \%$ vs. $3,6 \%)$ als auch im Liquor $(2,4 \%$ vs. $1,2 \%)$ deutlich häufiger auftraten als bei Patienten mit nichtentzündlichen neurologischen Erkrankungen. Allerdings waren die Liquorwerte für $\gamma \delta$ T-Zellen auch bei Patienten mit neurologischen Infekten oder anderen Autoimmunerkrankungen deutlich erhöht.

Spezifischer waren die Unterschiede, wenn man unter den $\gamma \delta$-T-Zellen nur den Anteil der Interleukin-17 produzierenden Zellen analysierte. Dieser Botenstoff wird bei MS auch von den autoreaktiven T-
Helferzellen freigesetzt. Der Anteil der IL-17 produzierenden $\gamma \delta$-T-Zellen im Liquor war mit $16,5 \%$ bei MS-Patienten und Patienten mit erstem MS-Schub am höchsten und deutlich niedriger bei Patienten mit neurologischen Infekten $(9,5 \%)$ oder mit anderen Immunerkrankungen oder nichtentzündlichen neurologischen Erkrankungen (6\%). Die Forscher schließen daraus, dass es bei MS zu einer spezifischen Anreicherung von IL-17 produzierenden $\gamma \delta$-T-Zellen im Liquor und ZNS kommt. Dies könnte für eine frühe Diagnose und Differenzialdiagnose wichtig sein und auch neue Ansatzpunkte für eine MS-Therapie bieten.

Thomas Müller

Schirmer L et al. Enriched CD $161^{\text {high }} \mathrm{CCR}^{+} \gamma \delta$ TCells in the Cerebrospinal Fluid of Patients With MS. Arch Neurol. Epub 2012, 17. Dezember; doi: 10.1001/2013.jamaneurol.409 